\title{
Rheumatoid Arthritis Therapy and Joint-replacement Surgery: Are We Making a Difference?
}

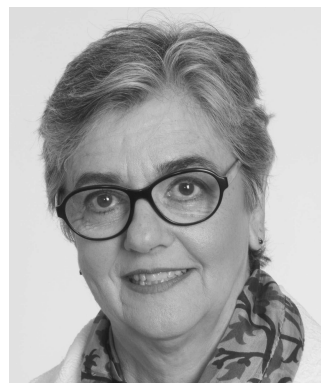

Outcomes for patients with rheumatoid arthritis (RA) have improved over the past decade, owing to recognition of the importance of a structured "treat-to-target" treatment protocol and of early treatment, in addition to increasing access to potent therapies. American College of Rheumatology response criteria, quality of life measures, and differences in radiographic progression have all been used as treatment endpoints to demonstrate improvement. Studies such as Grigor et al's TICORA and Verstappen et al's CAMERA clearly demonstrate that disease management protocols emphasizing tight control result in lower disease activity, more patients in remission, and less joint damage ${ }^{1,2}$. Initiation of therapy and achieving low disease activity early in the first year of disease has a major effect on clinical and radiographic outcomes years later ${ }^{3}$. Finally, initiating aggressive treatment after onset of RA and maintaining tight control is a feasible "real world" strategy 4 .

These changes provide the backdrop for reports that confirm a clear decrease in orthopedic surgery of the upper extremity and soft tissue for patients with rheumatoid arthritis (RA), although rates appear stable for total knee (TKA) and total hip arthroplasty (THA $)^{5,6}$. In this context, there is now greater interest in using rates of orthopedic surgery, and more specifically, arthroplasty as endpoints to indicate successful disease control in patients with RA.

In this issue of The Journal Widdifield and colleagues describe 2 cohorts of incident RA patients over 66 years of age in Ontario and Quebec, and investigate the relationship between the use of methotrexate (MTX) and other disease-modifying antirheumatic drug (DMARD) therapy and the rate of arthroplasty ${ }^{7}$. The outcome of interest was time from RA diagnosis to undergoing first arthroplasty. Data were obtained retrospectively from Canadian provincial health administrative databases that can boast fairly complete recording of healthcare events, including diagnosis, procedures, and drug use. Cases were validated as RA if there were 3 visits with the appropriate International Classification of
Diseases, 9th edition code for RA, including at least 1 visit to an internist or rheumatologist. Drug exposures were carefully calculated from pharmacy records. The main exposure of interest was MTX and other DMARD use, adjusting for other drug exposures including tumor necrosis factor inhibitors (TNFi). Demographics including socioeconomic status and comorbidities were obtained and used in the model. Using a Cox proportional hazard model measuring duration of drug use in the first year as a time-varying variable, they determined the effect of drug use on the hazard rate for arthroplasty.

The major finding of this study was that within the first year of diagnosis, duration of exposure to MTX in both Ontario (HR 0.97, 95\% CI 0.95-0.98) and Quebec (HR 0.96, 95\% CI 0.94-0.98), as well as duration of exposure to other DMARD (HR 0.98, 95\% CI 0.97-0.99), were independently associated with longer time to joint replacement, after adjustment for other covariates. Presented another way, their data demonstrate that use of MTX and other DMARD can decrease arthroplasty by $2 \%$ to $3 \%$ per month, for a maximal decrease in arthroplasty of $36 \%$ for a full year of MTX use in early RA. The investigators found few significant differences between the 2 cohorts, totaling 27,672 incident cases of RA, with 4.4 (Quebec) to 4.6 (Ontario) years of followup. In the combined 142,451 patient-years of followup for both cohorts, 437 (6.5\%, Quebec) and 2201 (10.5\%, Ontario) patients with RA underwent arthroplasty. The authors additionally noted that age, prior arthroplasty, comorbid osteoarthritis (OA) and nonsteroidal antiinflammatory use were risk factors for shorter time to arthroplasty.

These findings contribute to a growing body of literature asserting that early, continuous use of MTX and other synthetic DMARD in early RA delays joint replacement surgery. However, retrospective cohort studies using administrative databases such as this typically lack individual level data such as seropositivity or disease activity and severity, so data relevant to potential confounders may be missed,

See Joint replacements in RA, page 861

Personal non-commercial use only. The Journal of Rheumatology Copyright (C) 2016. All rights reserved. 
despite the inclusion of data on frequency of rheumatology visits, extraarticular disease, and corticosteroid use as proxy measures. Misclassification is another concern when administrative data are used; however, the authors apply previously described methods to improve the validity of the RA diagnosis, cumulative medication use, and specific procedures undertaken to minimize misclassification. In addition, the pharmacy claims database provides a relatively complete record for cumulative drug use for those over 66 years of age, which is the exposure of interest for this study, but younger patients could not be included. Use of biologics plays a very small role in this analysis, possibly owing to the difficulty in acquiring insurance approval for biologics during the first year of RA.

In contrast to the markedly increasing rates of arthroplasty for patients with OA, several recent studies report diminished or stable arthroplasty rates in patients with RA coinciding with the widespread use of biologics and DMARD ${ }^{6,8,9}$. However, the effect of medication use on arthroplasty rates or any other outcome is difficult to study directly, because the indications for medications and outcomes are typically interrelated in cohort studies, and prospective trials would be unreasonable both ethically and financially. Efforts include a study by Aaltonen and coworkers, who have reported on 2 cohorts of Finnish patients with RA. Surprisingly, patients receiving biologics had a higher incidence rate of arthroplasty than those receiving MTX and other DMARD, despite propensity score matching ${ }^{10}$. Similarly, Asai and colleagues, in a retrospective study of 155 TNFi-treated patients with RA, 79 of whom received concomitant MTX, found a significantly lower incidence of TKA in the MTX plus biologic-treated group ${ }^{11}$. Asai, et al pursued this question further using a multicenter registry, with 803 TNFi-treated patients with RA, and reported a significantly lower incidence of arthroplasty in the patients receiving MTX plus TNFi compared to those receiving TNFi alone ${ }^{12}$. Moura, et al also found that in the first year after RA diagnosis, longer exposure to MTX or other DMARD was associated with a longer time to arthroplasty ${ }^{13}$. Taken together, these studies raise the question whether early therapy in general, or more specifically, use of MTX and other traditional DMARD independent of biologics has a particular joint protective effect.

Another striking observation in the report of Widdifield, et al is the significant numbers of seniors who were not treated with MTX or other DMARD during the first year after diagnosis, suggesting that many patients with RA may not benefit from current standards of care. This raises important questions about the approach to treatment for patients with RA in the year after diagnosis. For the seniors included in this study, $74 \%-78 \%$ were treated with corticosteroids, and $23 \%-30 \%$ received no MTX or any other DMARD in the first year after diagnosis.

While an increased background prevalence of comor- bidities may lead to less aggressive RA therapy in seniors, there is ample published evidence demonstrating an increase in risk of infection with corticosteroid use in the elderly as well as evidence for their relative safety using MTX and DMARD $^{14,15}$. Others have also reported that age at the time of disease onset affects choice of therapy and that seniors receive less aggressive therapy ${ }^{16}$, despite the striking concordance that optimal therapy in RA includes DMARD therapy as soon as the diagnosis of RA is made ${ }^{17}$.

Improving the outcome of patients with RA is an ongoing effort, and goals now include a decreased need for major surgery such as arthroplasty. The findings of Widdifield and colleagues support the benefit of early use of MTX and DMARD in joint protection, add to the literature demonstrating the importance of treatment strategy on outcome, and raise the question of whether MTX and other DMARD can have a unique effect on joint preservation.

Equally important, the findings presented here suggest that too many patients with newly diagnosed RA are not receiving timely and appropriate therapy. To enhance the accuracy of pharmacy records in this study, all patients studied were 66 years and older, and therefore a younger comparator group could not be included, so we do not know whether seniors are disproportionately undertreated. This study raises important questions regarding delay in treatment of patients with RA, suggesting a potential area for intervention among seniors. More information is needed to better understand why some patients with early RA are still not receiving appropriate MTX or DMARD therapy.

\section{SUSAN M. GOODMAN, MD,}

Associate Professor of Clinical Medicine, Weill Cornell Medicine College, Associate Attending Physician, Department of Rheumatology, Hospital for Special Surgery, New York, New York, USA.

Address correspondence to Dr. S.M. Goodman, Hospital for Special Surgery, 535 East 70th St., New York, New York 10021, USA. E-mail: goodmans@hss.edu

\section{REFERENCES}

1. Grigor C, Capell H, Stirling A, McMahon AD, Lock P, Vallance R, et al. Effect of a treatment strategy of tight control for rheumatoid arthritis (the TICORA study): a single-blind randomised controlled trial. Lancet 2004;364:263-9.

2. Verstappen SM, Jacobs JW, van der Veen MJ, Heurkens AH, Schenk $\mathrm{Y}$, ter Borg EJ, et al. Intensive treatment with methotrexate in early rheumatoid arthritis: aiming for remission. Computer Assisted Management in Early Rheumatoid Arthritis (CAMERA, an open-label strategy trial). Ann Rheum Dis 2007;66:1443-9.

3. Aletaha D, Alasti F, Smolen JS. Optimisation of a treat-to-target approach in rheumatoid arthritis: strategies for the 3-month time point. Ann Rheum Dis 2015 Sep 29 (E-pub ahead of print).

4. Haraoui B, Bensen W, Bessette L, Le Clercq S, Thorne C, Wade J. Treating rheumatoid arthritis to target: a Canadian physician survey. J Rheumatol 2012;39:949-53.

5. Nikiphorou E, Carpenter L, Morris S, Macgregor AJ, Dixey J, Kiely

Personal non-commercial use only. The Journal of Rheumatology Copyright $\odot$ 2016. All rights reserved. 
$\mathrm{P}$, et al. Hand and foot surgery rates in rheumatoid arthritis have declined from 1986 to 2011, but large-joint replacement rates remain unchanged: results from two UK inception cohorts. Arthritis Rheumatol 2014;66:1081-9.

6. Sokka T, Kautiainen H, Hannonen P. Stable occurrence of knee and hip total joint replacement in Central Finland between 1986 and 2003: an indication of improved long-term outcomes of rheumatoid arthritis. Ann Rheum Dis 2007;66:341-4.

7. Widdifield J, Moura CS, Wang Y, Abrahamowicz M, Paterson JM, Huang AA, et al. The longterm effect of early intensive treatment of seniors with rheumatoid arthritis: a comparison of 2 population-based cohort studies on time to joint replacement surgery. J Rheumatol 2016;43:861-8.

8. Mertelsmann-Voss C, Lyman S, Pan TJ, Goodman SM, Figgie MP, Mandl LA. US trends in rates of arthroplasty for inflammatory arthritis including rheumatoid arthritis, juvenile idiopathic arthritis, and spondyloarthritis. Arthritis Rheumatol 2014;66:1432-9.

9. Momohara S, Inoue E, Ikari K, Kawamura K, Tsukahara S, Iwamoto $\mathrm{T}$, et al. Decrease in orthopaedic operations, including total joint replacements, in patients with rheumatoid arthritis between 2001 and 2007: data from Japanese outpatients in a single institute-based large observational cohort (IORRA). Ann Rheum Dis 2010; 69:312-3.

10. Aaltonen KJ, Virkki LM, Jamsen E, Sokka T, Konttinen YT, Peltomaa R, et al. Do biologic drugs affect the need for and outcome of joint replacements in patients with rheumatoid arthritis? A register-based study. Semin Arthritis Rheum 2013;43:55-62.

11. Asai S, Takahashi N, Funahashi K, Yoshioka Y, Takemoto T, Terabe $\mathrm{K}$, et al. Concomitant methotrexate protects against total knee arthroplasty in patients with rheumatoid arthritis treated with tumor necrosis factor inhibitors. J Rheumatol 2015;42:2255-60.

12. Asai S, Kojima T, Oguchi T, Kaneko A, Hirano Y, Yabe Y, et al. Effects of concomitant methotrexate on large joint replacement in patients with rheumatoid arthritis treated with tumor necrosis factor inhibitors: A multicenter retrospective cohort study in Japan. Arthritis Care Res 2015 (E-pub ahead of print).

13. Moura CS, Abrahamowicz M, Beauchamp ME, Lacaille D, Wang Y, Boire G, et al. Early medication use in new-onset rheumatoid arthritis may delay joint replacement: results of a large population-based study. Arthritis Res Ther 2015;17:197.

14. Dixon WG, Abrahamowicz M, Beauchamp ME, Ray DW, Bernatsky S, Suissa S, et al. Immediate and delayed impact of oral glucocorticoid therapy on risk of serious infection in older patients with rheumatoid arthritis: a nested case-control analysis. Ann Rheum Dis 2012;71:1128-33.

15. Bologna C, Viu P, Jorgensen C, Sany J. Effect of age on the efficacy and tolerance of methotrexate in rheumatoid arthritis. $\mathrm{Br} \mathrm{J}$ Rheumatol 1996;35:453-7.

16. Innala L, Berglin E, Moller B, Ljung L, Smedby T, Sodergren A, et al. Age at onset determines severity and choice of treatment in early rheumatoid arthritis: a prospective study. Arthritis Res Ther 2014;16:R94

17. Singh JA, Saag KG, Bridges SL Jr, Akl EA, Bannuru RR, Sullivan MC, et al. 2015 American College of Rheumatology Guideline for the Treatment of Rheumatoid Arthritis. Arthritis Rheumatol 2016;68:1-26.

J Rheumatol 2016;43:833-5; doi:10.3899/jrheum.160043 